\title{
Intrinsic Absorption and Emission Lines in Seyfert Galaxies
}

\author{
D. M. Crenshaw
}

Computer Sciences Corporation, Laboratory for Astronomy and Solar Physics, NASA Goddard Space Flight Center, Code 681, Greenbelt, MD 20771, USA

\begin{abstract}
Recent $H S T$ spectra indicate that a large fraction of Seyfert 1 galaxies have UV absorption lines that are intrinsic to the active nucleus or its environment. IUE monitoring or multiple $H S T$ observations of several sources show that many absorption lines are variable, either as a result of mass motion across the line-of-sight or of changing ionization within the gas in response to variations in the ionizing continuum. The UV absorbers may be related to the warm absorbers seen in the X-ray spectra of many Seyfert galaxies, but a direct connection between the two has not been made. Some of the absorption lines may arise in the emission-line regions in the nucleus or host galaxy, providing valuable clues to their nature.
\end{abstract}

\section{Intrinsic Absorption - A Common Occurrence}

Prior to the launch of $H S T$, we knew that the UV spectrum of a Seyfert 1 galaxy would show a strong nonthermal continuum from the nucleus, emission lines from the surrounding broad-line region (BLR) and narrow-line region (NLR), and absorption lines from our own Galaxy. Only a small percentage $(\sim 3 \%)$ of $I U E$ observations show evidence for narrow absorption lines that originate near the redshift of the active galaxy (Ulrich 1988). However, only the strongest absorption lines (equivalent widths $>1 \AA$ ) can be detected in IUE spectra, due to the low signal-to-noise and resolution of the IUE data. Our FOS and GHRS spectra of a small sample of Seyfert 1 galaxies indicate that the incidence of intrinsic absorption lines is much higher than $3 \%$, and may be closer to $50 \%$.

\section{Unanswered Questions}

A number of questions concerning the importance of the UV absorption lines arise, and some preliminary answers can be given:

1. Do they arise from the warm absorbers seen in the X-rays? The evidence at this point is circumstantial. An ionization parameter of $U=1-10 \mathrm{can}$ produce the $\mathrm{O}$ VII and $\mathrm{O}$ VIII absorption edges and the column densities of $\mathrm{C} I V$ and $\mathrm{N} \vee$ in a few sources (Mathur 1994; Mathur et al. 1994). However, in some cases (e.g., NGC 3516), the column density of C IV is too high to be consistent with a single-zone model. 
2. Are they related to the 'associated absorption' or broad absorption lines in QSOs? Again, the connection is uncertain, but we note that a number of $0.3 \leq z \leq 1.0$ QSOs observed with the FOS (Bahcall et al. 1993) show blue-shifted absorption lines similar to those seen in our Seyfert spectra.

3. What is their relation to the intrinsic emission lines? The origin of the absorption lines, and their connection to the emission lines, is a mystery. However, even the highly ionized warm absorber is expected to produce some observable emission, particularly in the lines of Ne vill $\lambda 774$, $\mathrm{O}$ VI $\lambda 1035$, and Ly $\alpha$ (Hamann et al. 1995). Obviously, if the UV absorption lines are linked to the emission-line regions, they can provide valuable constraints on column densities, covering factors, and radial velocities in the line-of-sight.

4. What is the source of their variability? Is it bulk motion of gas across the line-of-sight or variable ionization? It seems that whenever multiple observations of a source have been obtained at sufficient signal-to-noise and resolution, the UV absorption lines have varied. Recently, we discovered variable C IV absorption with two GHRS spectra of NGC 3783 obtained 11 months apart (Maran et al. 1996). In addition, there is strong evidence from IUE monitoring that the time scale for variations can be as short as weeks or possibly even days (Bromage et al. 1985; Voit et al. 1987). Variable ionization of the gas is a more reasonable explanation for the short time-scale variations (Maran et al. 1996), but there is some evidence for bulk motion on a time scale of years in NGC 3516 (Walter et al. 1990).

If the source of variability is bulk motion, the monitoring observations can determine the transverse velocities and changes in radial velocity flow. If the variability is due to changing ionization in response to variations in the ionizing continuum, then monitoring can determine the recombination time, which yields the electron number density. From the density and ionization parameter, the distance of the absorber from the continuum source can be determined.

\section{Preliminary Results}

The existing observations are summarized in Table 1, which lists the Seyfert galaxies that have $H S T$ spectra with sufficiently good signal-to-noise and resolution in the UV, and $A S C A$ spectra that can be used to detect a warm absorber (Mushotzky, George, \& Turner 1995). The small sample in Table 1 suggests that there is a correlation between the UV and X-ray absorbers. However there are two Seyfert galaxies that do not fit the pattern: Mrk 335 and Mrk 509.

The intrinsic absorption lines in the $H S T$ spectra have a number of similar properties:

1. They are all blueshifted by zero to $-1500 \mathrm{~km} \mathrm{~s}^{-1}$, which indicates net radial outflow.

2. Many of the CIV absorption components are moderately broad (100$300 \mathrm{~km} \mathrm{~s}^{-1}$ ), indicating macroscopic motions, since a temperature of $T=$ $10^{5} \mathrm{~K}$ would produce a thermal width of only $20 \mathrm{~km} \mathrm{~s}^{-1}$ FWHM. In addition, multicomponent profiles are common. 
Table 1. Seyfert 1 galaxies with $A S C A$ and $H S T$ spectra.

\begin{tabular}{|c|c|c|c|c|c|c|c|}
\hline Name & $\bar{z}$ & Warm Abs? & UV Abs? & Name & $z$ & Warm Abs? & UV Abs? \\
\hline NGC 3783 & 0.009 & Yes & Yes & Mrk 335 & 0.025 & Yes? & No \\
\hline NGC 5548 & 0.017 & Yes & Yes & Mrk 509 & 0.035 & No & Yes \\
\hline NGC 4151 & 0.003 & Yes & Yes & Fairall 9 & 0.046 & No & No \\
\hline NGC 3516 & 0.009 & Yes & Yes & Akn 120 & 0.033 & No & No \\
\hline
\end{tabular}

3. High-ionization lines (e.g., C IV $\lambda \lambda 1548,1550 ; \mathrm{N} v \lambda \lambda 1238,1242$ ) are always present. The $\mathrm{O}$ VI $\lambda \lambda 1032,1038$ lines have been detected in HUT spectra of NGC 4151 (Kriss et al. 1992) and NGC 3516 (Kriss et al. 1996). Lowionization lines, such as $\mathrm{Mg}$ II $\lambda \lambda 2796,2803$, have not been seen, except in the case of NGC 4151 (Bromage et al. 1995).

4. As mentioned earlier, the absorption lines are variable on time scales of weeks to years, and possibly days. There is evidence for both variable ionization and bulk motion in NGC 3516 (Walter et al. 1990).

5. The cores of the strongest absorption lines are deeper than the continuum heights. In some case, the absorption lines go to zero intensity, which indicates that the absorber is completely outside of the broad emissionline region.

6. Comparing different objects, there is a large range in $\mathrm{C}$ IV column density; for example $N(\mathrm{C}$ IV $) \approx 10^{14} \mathrm{~cm}^{-2}$ in $N G C 3783$ and $N\left(\mathrm{C}_{\mathrm{IV}}\right) \approx 10^{18} \mathrm{~cm}^{-2}$ in NGC 3516. The former is at least consistent the column densities expected from a single-zone warm absorber (Shields \& Hamann 1996), but the latter is not.

Figure 1 shows a recent GHRS spectrum of NGC 3516 (Crenshaw et al. 1996), which exhibits many of the properties described above. There are four discernible radial velocity components of the $\mathrm{C}$ IV doublet; the fourth component is very close to the redshift of the host galaxy.

\section{Future Observations}

Intrinsic absorption lines appear in a large fraction of low-luminosity AGN; this fact alone suggests that they will be valuable in unraveling the complicated structure of AGN. If the absorption lines are found to be associated with the warm absorber and/or emission-line regions, they will also yield important constraints on photoionization and kinematic models of these regions. More data are needed in the following categories:

1. Low-resolution ( $R \equiv \lambda / \Delta \lambda \approx 1000$ ) UV spectra to increase the sample of AGN with known intrinsic absorbers, determine the range of absorber properties, covering factors, and for statistical correlations with other AGN properties (e.g., luminosity). 


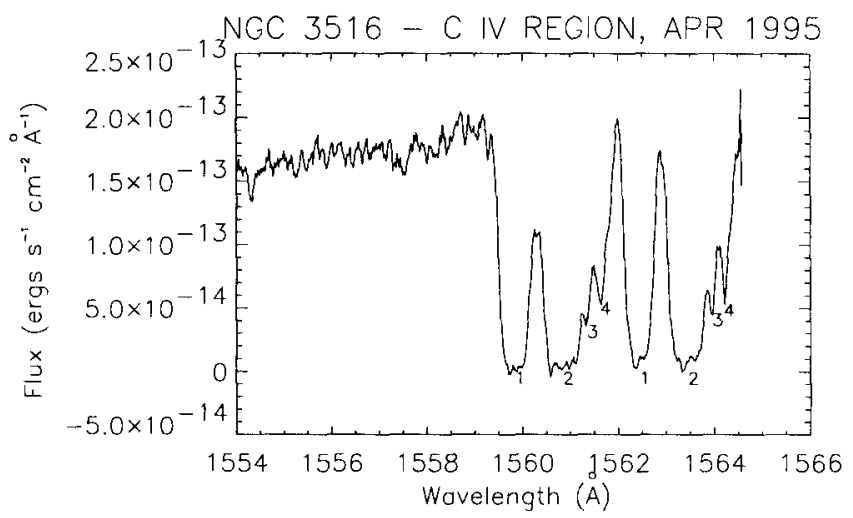

Figure 1. Expanded GHRS spectrum of NGC 3516 in the core of the $\mathrm{C}$ IV emission line. CIV doublets in absorption at four distinct radial velocities are noted.

2. High-resolution $(R \approx 10,000)$ UV spectra to deconvolve the absorptionline components, determine accurate column densities, and study the velocity structure.

3. Simultaneous UV and X-ray monitoring to determine the causes of variability (variable ionization and/or bulk motion), and the connection to warm absorbers.

\section{References}

Bahcall, J. N., et al. 1993, ApJ, 87, 1.

Bromage, G. E., et al. 1985, MNRAS, 215, 1.

Crenshaw, D. M., et al. 1996, in preparation.

Hamann, F., Shields, J. C., Ferland, G. J., \& Korista, K. T. 1995, ApJ, 454, 688.

Kriss, G. A., Davidsen, A. F., Zheng, W., Kruk, J. W., \& Espey, B. R. 1995, ApJ, $454, \mathrm{~L} 7$.

Kriss, G. A., et al. 1996, preprint.

Maran, S. P., et al. 1995, ApJ, 465, 733.

Mathur, S. 1994, ApJ, 431, L75.

Mathur, S., Elvis, M., \& Wilkes, B. 1995, ApJ, 452, 230.

Mushotzky, R., George, I., \& Turner, J. 1995, private communication.

Shields, J. C., \& Hamann, F. 1996, preprint.

Ulrich, M.-H. 1988, MNRAS, 230, 121.

Voit, G. M., Shull, J. M., \& Begelman, M. C. 1987, ApJ, 316, 573.

Walter, R., Ulrich, M.-H., Courvoisier, T. J.-L., \& Buson, L. M. 1990, A\&A, 233, 53. 